\title{
Sex-specific cardiac cardiolipin remodelling after doxorubicin treatment
}

\author{
Maryline Moulin ${ }^{1,5^{*}}$, Audrey Solgadi ${ }^{2,3}$, Vladimir Veksler $^{1}$, Anne Garnier ${ }^{1}$, Renée Ventura-Clapier ${ }^{1}$ \\ and Pierre Chaminade $2,3,4$
}

\begin{abstract}
Background: Imbalance in lipid metabolism and membrane lipid homeostasis has been observed in numerous diseases including heart failure and cardiotoxicity. Growing evidence links phospholipid alterations especially cardiolipins (CLs) to defects in mitochondrial function and energy metabolism in heart failure. We have shown recently that doxorubicin cardiotoxicity is more severe in male than female Wistar rats. We aimed to study whether this sex specificity is linked to differences in cardiac phospholipid profiles.

Results: Adult male and female rats were injected $2 \mathrm{mg} / \mathrm{kg}$ doxorubicin weekly for 7 weeks. Cardiac phospholipid molecular species were determined by liquid chromatography coupled with mass spectrometry fragmentation (LC)/ $M^{n}$. Sex difference in phosphatidylethanolamine and phosphatidylcholine species containing docosahexaenoic and docosapentaenoic acyl chains was observed, females having more than males. In both sexes, doxorubicin induced an important loss of the main $\mathrm{CL}(18: 2)_{4}$, while the level of monolysocardiolipin $\mathrm{MLCL}(18: 2)_{3}$ remained stable. However, a severe remodelling appeared in treated rats with the longest $\mathrm{CL}$ acyl chains in doxorubicin-treated females, which might compensate for the loss of tetra-linoleoyl CL. The level of oxidized cardiolipin was not particularly increased after doxorubicin treatment. Finally, expression of genes involved in the biosynthesis of fatty acid appeared to be decreased in doxorubicin-treated males.
\end{abstract}

Conclusions: These results emphasize for the first time the cardiac remodelling in the phospholipid classes after doxorubicin treatment. These observations suggest that doxorubicin has a sex-specific impact on the heart phospholipidome especially on cardiolipin, an essential mitochondrial lipid. Further studies are needed to better understand the roles of lipids in the anthracycline cardiotoxicity and sex differences, but phospholipid cardioprotection seems a valuable new additive therapeutic strategy for anthracycline cardiotoxicity.

Keywords: Cardiolipin, Sex-specific, Heart failure, Doxorubicin, LC/MS

\section{Background}

Doxorubicin (DOXO), a widespread anti-cancer agent, has clinical limited use due to important deleterious cardiac side effects that ultimately lead to heart failure (HF). How anthracyclines induce both anti-cancer activity and cardiotoxicity are not completely understood [1]. Numerous studies have shown the interaction of doxorubicin with membranes, particularly cardiolipin (CL), causing membrane fluidity alteration and enzyme activity

\footnotetext{
* Correspondence: maryline.moulin@univ-paris-diderot.fr

'UMR-S 1180, Inserm, Univ Paris-Sud, Université Paris-Saclay,

Châtenay-Malabry, France

${ }^{5}$ Current address: Université Paris Diderot, Unité de Biologie Fonctionnelle et Adaptative, CNRS UMR 8251, Paris, France

Full list of author information is available at the end of the article
}

deficiency [2-4]. We have recently observed that after 7 weeks of doxorubicin treatment, male rats but not females had high mortality rate, signs of heart failure, and altered mitochondrial function, and we identified a significant decrease in total cardiolipin content specifically in the heart of treated males [5].

Phospholipids (PLs), a fundamental class of biological compounds, are the main component of membranes which represents a biological and physical barrier but also a site for interaction and signal transduction. Fatty acid (FA) composition of PL influences membrane characteristics such as membrane fluidity, microdomain formation, transport system, and membrane-bound enzyme activities (for review, see [6]). Obviously, deregulation of PL is involved in numerous diseases such as cancer, neurological 
disorders, hepatic disorders, and cardiovascular diseases (reviewed in $[7,8]$ ).

Cardiolipin, a signature PL of the inner membrane of the mitochondria (around $20 \%$ of the lipid composition), is involved in numerous functional and structural features of membrane-bound mitochondrial proteins and is linked to important mitochondrial processes, including apoptosis, mitochondrial dynamics, contact sites formation and assembly, and function of mitochondrial membrane proteins for an effective oxidative phosphorylation (reviewed in [9-12]). CL is a unique dimeric phospholipid containing two phosphatidyl moieties bridged by a glycerol and four fatty acyl chains. The FA composition is also highly specific and is predominantly composed of 18:2 acyl chains with tetra-linoleoyl cardiolipin $\left(\mathrm{L}_{4} \mathrm{CL}\right)$ being the most abundant [13]. CL composition depends on de novo synthesis of nascent CL followed by complex processes of acyl chain remodelling. The fatty acyl chain composition of $\mathrm{CL}$ is critical for mitochondrial function and cardiac pathology. Alteration in CL content and composition has been associated with numerous disorders like diabetes, thyroid status, aging, and heart failure [14].

HF is characterized by the inability of the heart to fulfill the metabolic requirements of the organism. Among the characteristics of HF, the state of energy depletion $[15,16]$, alterations of the mitochondrial function, and biogenesis [17] have been well described, but changes in cardiac PL composition are less addressed. However, cardiolipin may play a role in mitochondrial alterations in heart failure [10]. Indeed, decreased cardiolipin content, altered cardiolipin synthesis and remodelling [18], reduction in 18:2 CL species [10,18, 19], and modified expression or activity of cardiac biosynthesis enzymes [20] have been reported in numerous diseases. The importance of CL in heart failure is exemplified by the Barth syndrome, a severe $\mathrm{X}$-linked genetic disorder which is characterized by $\mathrm{CL}$ alterations, mitochondrial defects, and heart failure and which results from mutations in the Tafazzin gene, an acyltransferase involved in CL remodelling [21]. Finally, CLs are susceptible to oxidative damages because they are present in the mitochondria which are the main source of reactive oxygen species (ROS) and because they contain double bonds that are susceptible to oxidation. CL oxidation has been shown to induce decreased activity and organisation of respiratory chain complexes and to favor apoptosis (for review, see [11]).

As we have observed, a decrease in total cardiolipin content in treated males but no females; the objective of the present study was thus to explore in more detail the cardiac phospholipidome after doxorubicin treatment in adult rats and to understand whether $\mathrm{CL}$ remodelling participates in the sex-specific cardiac alterations.

\section{Methods}

An expanded method section is available in the Additional file 1.

\section{Doxorubicin animal model}

A chronic model of doxorubicin [22] by weekly intravenous injection of $2 \mathrm{mg} / \mathrm{kg}$ doxorubicin or saline solution during 7 weeks in 11-week-old male and female Wistar rats (Janvier Labs) was chosen as previously described [23]. Animal experimental procedures were approved by the Animal Ethics Committee of Paris Sud University. Investigations were done in accordance with European Community legislation relating to the care and use of animals (Directive 2010/63/EU) and the corresponding French legislation (French decree 2013-118 du 1er février 2013).

\section{Lipid extraction}

A Folch method [24] was used to extract the lipids from 10 to $20 \mathrm{mg}$ of heart homogenized in phosphate-buffered saline (PBS). Each group was composed of four animals; one technical repeat was done for a non-treated male and a doxorubicin-treated male. Total lipids were extracted by adding $1.5 \mathrm{~mL}$ of methanol and $3 \mathrm{~mL}$ of chloroform to the tissue suspension. After centrifugation at $1000 \mathrm{~g}$ for $10 \mathrm{~min}$, the lower phase containing total lipids was collected and evaporated to dryness at room temperature under nitrogen gas. The samples were resuspended in $100 \mu \mathrm{L}$ of chloroform per $10 \mathrm{mg}$ of heart and subsequently analyzed.

\section{Cardiolipin oxidation}

Cardiolipins were oxidized following the method of Paradies [25]. Briefly, cardiolipins in PBS were mixed in $1 \mathrm{~mL}$ of oxygenated buffer containing $20 \mu \mathrm{M}$ ferrous sulfate, $120 \mu \mathrm{M}$ ADP, and $200 \mu \mathrm{M}$ ascorbic acid for $15 \mathrm{~min}$ at $37{ }^{\circ} \mathrm{C}$ before being extracted by a Folch method.

\section{Phospholipid analysis}

For the phospholipid analysis, each sample was duplicated, except the technical repeat for a non-treated male. Chromatographic system from Thermo Fisher Scientific included a Dionex U 3000 RSLC system with two quaternary pumps, an autosampler, and a column oven. The RSLC system was coupled online to a charged aerosol detector Corona CAD Ultra for the quantitative part of the study and a LTQ Orbitrap Velos Pro for the lipidomic study (all from Thermo Fisher).

\section{Liquid chromatography}

The separation of lipids was carried out on a PVA-Sil column $(150 \times 2.1$ mm I.D., 120 A) (YMC Europe GmbH) 
with a $10 \times 2 \mathrm{~mm}$ guard column packed with the same material both supplied by Interchim. The column temperature was thermostatically controlled at $35{ }^{\circ} \mathrm{C}$. Chromatographic method was inspired from the method developed by Imbert et al. [26]. The flow rate was set at $400 \mu \mathrm{L} / \mathrm{min}$, and a sample volume of $5 \mu \mathrm{L}$ was injected. The mobile phase compositions were identical. An isopropanol phase (D) has been added to rinse the RSLC system. The solvent program has been modified as follows: the Corona CAD nebulizer was set at a temperature of $30{ }^{\circ} \mathrm{C}$ and the nitrogen pressure was set at 5 bar (Table 1).

The LC effluent was split after the analytical column with aid of a tee; thus, $250 \mu \mathrm{L} / \mathrm{min}$ entered to the Corona $\mathrm{CAD}$ and $150 \mu \mathrm{L} / \mathrm{min}$ entered the mass spectrometer. To increase the flow entering the Corona CAD and maintain good aerosol stability, absolute ethanol was added with a second mixing tee with a flow of $200 \mu \mathrm{L} / \mathrm{min}$.

\section{Mass spectrometry}

The LTQ Orbitrap Velos Pro is equipped with an H-ESI II probe. Spray voltage was set at $3.3 \mathrm{kV}$. Heater temperature of the probe was set at $200{ }^{\circ} \mathrm{C}$. Sheath gas, auxiliary gas, and sweep gas flow rates were set at 20, 8, and 0 (arbitrary unit), respectively. Capillary temperature was set at $325{ }^{\circ} \mathrm{C}$ and S-lens RF level at $60 \%$. Analysis was performed in negative mode to obtain structural nformation on phospholipids fatty chains. This mass spectrometer is equipped with two analyzers: a double linear ion trap (LTQ) for fragmentation at low resolution and an orbital trap for high-resolution detection. The detection was performed either in full mass spectrometry (MS) scan with 100,000 resolution and data dependant $\mathrm{MS}^{2}$ and $\mathrm{MS}^{3}$ with collision-induced dissociation in the CID fragmentation (collision energy set at 35).

Table 1 Chromatographic program with the following mobile phase compositions: mixture of heptane and isopropanol 98:2 $(\mathrm{v} / \mathrm{v})$ (phase A); mixture of chloroforme and isopropanol 65:35 $(\mathrm{v} / \mathrm{v})$ (phase B); mixture of methanol and water 95:5 ( $/ \mathrm{v}$ ) (phase C); isopropanol (phase D)

\begin{tabular}{lllll}
\hline Time & $A$ & $B$ & $C$ & $D$ \\
0 & 98 & 2 & 0 & 0 \\
2 & 98 & 2 & 0 & 0 \\
8 & 12 & 88 & 0 & 0 \\
22 & 0 & 60 & 40 & 0 \\
26 & 0 & 60 & 40 & 0 \\
27 & 0 & 0 & 0 & 100 \\
29 & 0 & 0 & 0 & 100 \\
30 & 98 & 2 & 0 & 0 \\
44 & 98 & 2 & 0 & 0 \\
\hline
\end{tabular}

All $\mathrm{A}, \mathrm{B}$ and $\mathrm{C}$ mobile phases also contain acid acetic $1 \%(\mathrm{v} / \mathrm{v})$ and triethylamine $0.08 \%(\mathrm{v} / \mathrm{v})$

\section{Phospholipid species identification}

The chromatographic method used separates the phospholipids by polar head. Retention time is a crucial information for polar head identification and compared to commercial standards. Then, species can be observed under each chromatographic peak. PL identification was performed as described [27] using high-resolution mass detection in full scan mode and $\mathrm{MS}^{2} / \mathrm{MS}^{3}$ fragmentation in data dependant mode. $\mathrm{MS}^{\mathrm{n}}$ fragmentation has not always been performed by the instrument due to very small amount of some ions. When no MS ${ }^{\mathrm{n}}$ data is available, PLs are mentioned as, e.g., PL(34:1). Otherwise, PL are mentioned as, e.g., $\mathrm{PL}(16: 0-18: 1)$. Sn1 and sn2 position of the acyl chains were not determined. Oxidized cardiolipin identification was performed as described by Kim et al. [28, 29].

\section{Phospholipid species representation}

Total phospholipid amount was determined with Corona CAD detection by calculating the concentration of each PL class (in micrograms per milliliter) compared to calibration ranges of commercial standards. PL species intensities were determined by taking the mean relative intensity of $\mathrm{m} / \mathrm{z}$ ions over the chromatographic peak of each PL class.

\section{$2 D$ separation of $C L$}

The sample's cardiolipin was collected from 11 to $12 \mathrm{~min}$, dried over nitrogen, and dissolved in 2D phase mobile (2propanol:water:triethylamine:acetic acid 45:5:0.25:0.25). A Dionex C18 column (Acclaim RSLC 120, $2.2 \mu \mathrm{m}, 120 \AA$, $2.1 \times 150 \mathrm{~mm}$ ) was used under isocratic solvent condition. The column temperature was thermostatically controlled at $40{ }^{\circ} \mathrm{C}$. Flow was maintained at $200 \mu \mathrm{L} / \mathrm{min}$ on a Dionex U 3000 RSLC system, and MS and MS/MS acquisitions via LTQ Orbitrap Velos Pro were performed on the most abundant mass ions.

\section{Statistical analysis}

MS raw data were submitted to chromatogram builder, alignment and gap filling using the MzMine 2.10 LC/MS toolbox [30]. SIMCA-P 12.0 was used to analyze the data generated by mass spectrometry (principal component analysis (PCA) and OPLS-DA). Data are expressed as mean $\pm \mathrm{SEM}$. Two-way ANOVA (homogenous variance verified by Levene test for all parameters except $C d s 1$ and Acsl1) was used to study the global effect of sex and treatment, followed by StudentNewman-Keuls post hoc test to determine individual differences. Statistical significance for doxorubicin effect was defined as * for $p<0.05$, * for $p<0.01$, and ${ }^{* * *}$ for $p<0.001$ and for sex effect as $\mathbb{S}$ for $p<0.05$, $\mathbb{S S}$ for $p<0.01$, and $\mathbb{S S S}$ for $p<0.001$. 


\section{Results}

Doxorubicin treatment affects cardiac phospholipid content differently between males and females

Doxorubicin treatment induces a life-threatening cardiotoxicity with altered mitochondrial function. We have shown recently that males were less protected against DOXO cardiotoxicity and that cardiolipin content was differently altered in males and females under DOXO. To evaluate the cardiac phospholipid profile in doxorubicin-mediated cardiotoxicity, we performed lipidomic analysis of the hearts from male and female rats treated or not with doxorubicin for 7 weeks. To separate PL classes, we used a PVA-Sil column with a gradient program, used CAD for PL quantification and an ESI hybrid mass spectrometer (ESI/MS) to characterize the PL (Fig. 1a). The level of pooled main phospholipids (CL/phosphatidylinositol (PI)/phosphatidylethanolamine (PE)/phosphatidylserine (PS)/phosphatidylcholine (PC)) was similar in basal condition for both sexes; however, after doxorubicin treatment, cardiac phospholipid content was decreased only for the males (Fig. 1b). Principal component analysis of variables from LC-MS/MS analysis

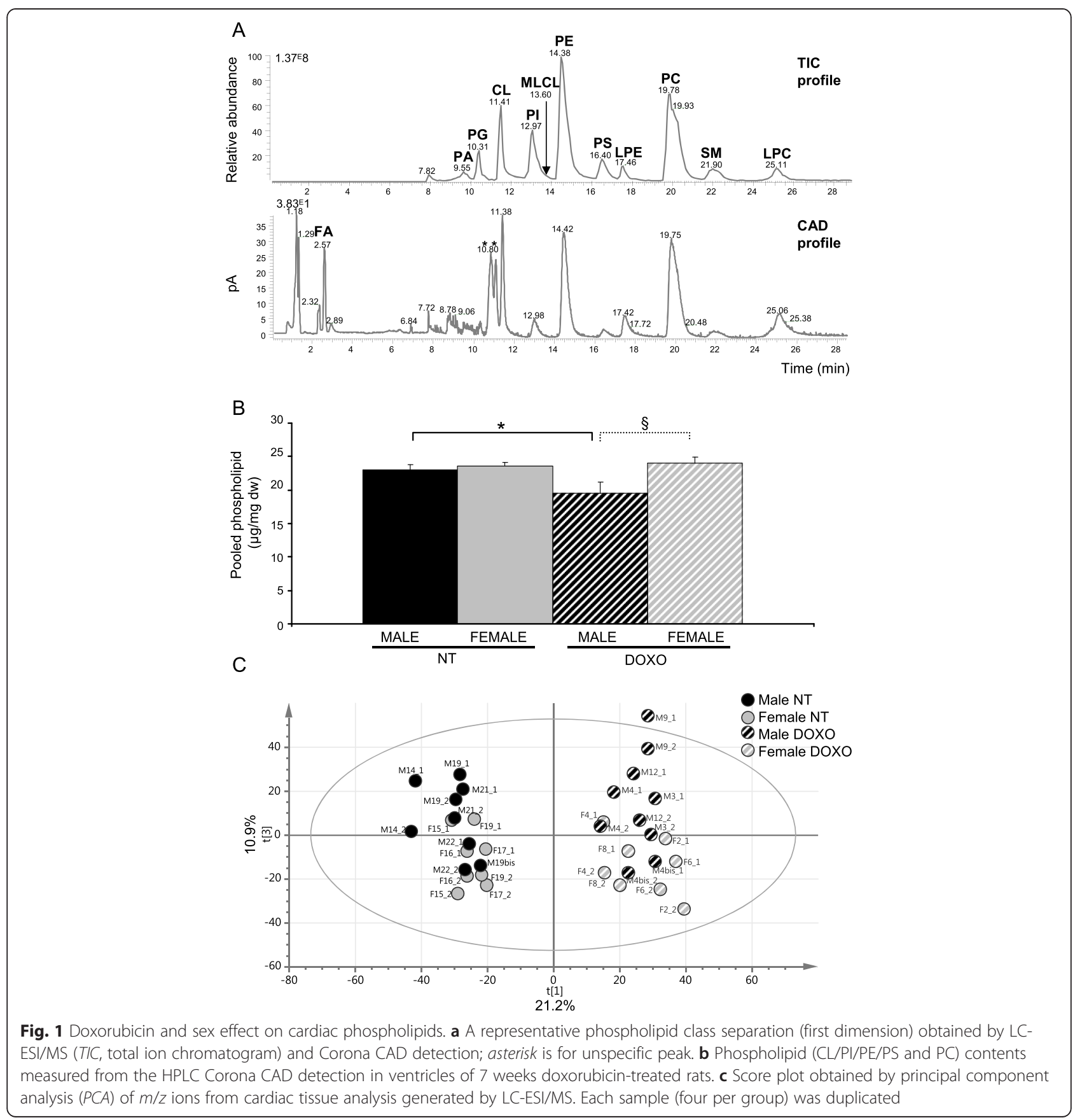


showed that non-treated animals clearly separate from doxorubicin-treated animals ( $21.2 \%$ of variance explained on principal component PC1), and males trend to separate from females (10.9\% of variance explained on PC3) (Fig. 1c). These analyses are in accordance with our previous results demonstrating sex difference in DOXO cardiotoxicity [5]. The MS data were then subjected to orthogonal partial least squares discriminant analysis (OPLS-DA) in order to detect the variables which characterise the most important changes between male and female in non-treated condition or after doxorubicin treatment and the effect of treatment in each sex
(Additional file 1: Table S1). We found mainly discriminating PE and CL species and less species of PC, phosphatidylglycerol (PG), and monolysocardiolipin (MLCL). Of note was the number of PL species with longer chains ( $\geq 20$ carbons) showing sex differences. These results underline variation of cardiac phospholipid composition after doxorubicin treatment and differently according to sex.

As PE and PC are the most abundant cardiac phospholipids (Fig. 1a), we then analyzed in more detail their fatty acyl chain composition. As shown in Fig. 2a, important sex differences are present at baseline and after DOXO for

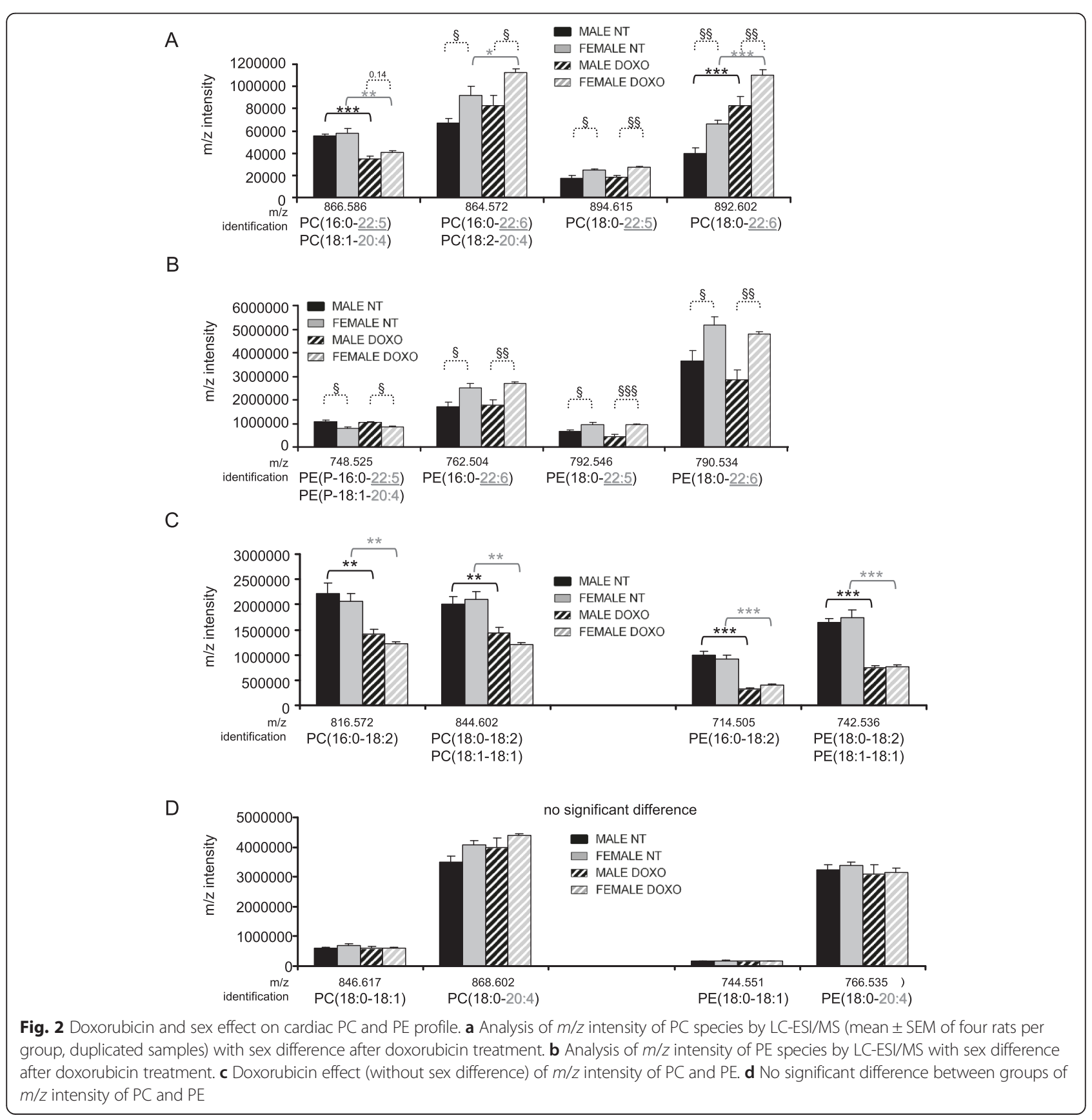


PC containing docosapentaenoic (22:5) and docosahexaenoic (22:6) acyl chains. Interestingly, the same profile was observed for the PE species with similar acyl chains (Fig. 2b). In contrast, PE and PC consisting of linoleoyl acyl chain (18:2) combined with saturated palmitic or stearic acyl chain did not present sex difference but were strongly reduced by DOXO treatment in both sexes (Fig. 2c). Finally, two other PE and PC species presented no change among sex and treatment (Fig. 2d). These results underline a great variability of $\mathrm{PE}$ and $\mathrm{PC}$ species and show that the level of the different species may vary according to doxorubicin and/or sex.

\section{Sex-specific cardiolipin remodelling after doxorubicin treatment}

As we previously showed, a decrease in CL content particularly in DOXO-treated males [5], analysis of cardiolipin species was necessary to dissect this deficit. Because of the four acyl chains, a great variety of CL was identified (Additional file 1: Table S2). A marked reduction of the most abundant CL: the tetra-linoleoyl $(18: 2)_{4}\left(\mathrm{~L}_{4} \mathrm{CL}\right)$ was observed after DOXO treatment but with no differences between sex (Fig. 3a). In contrast, almost all the other CL species were more abundant after DOXO treatment, and even more for the females (Fig. 3b), which may compensate for the tetra-linoleoyl CL loss. Doxorubicin induced a profound remodelling of $\mathrm{CL}$ species with longer acyl chains ( $\geq 20$ carbons). Some cardiolipin species were detected only after doxorubicin (Fig. 3c). Moreover, sex difference occurred only after doxorubicin treatment, as no basal male-female difference was observed (Fig. $3 \mathrm{a}-\mathrm{c}$ ). These results showed the complexity of CL profile in the heart. As summarized in the Table 2, the cardiotoxic treatment induced a severe remodelling in the CL family and the formation of new CL species and differentially between sex, as shown by the interaction between sex and doxorubicin treatment specific for cardiolipin.

\section{Low level of oxidized cardiolipin after 7 weeks of doxorubicin treatment in males}

Because cardiolipin oxidation has been observed in different pathologies and doxorubicin is a well-known stress oxidant inducer, we analyzed CL oxidation. Based on a previous study [31], we performed a twodimensional HPLC coupled to MS to separate oxidized CL from non-oxidized counterparts. As a positive control, we oxidized standard CLs (CLox) with oxygenated buffer containing ferrous sulfate, ADP, and ascorbic acid. We verified that the CLox were part of the cardiolipin peak in the first dimension (Additional file 1: Figure S1A). In the second dimension, CLox were resolved into multiple peaks (Additional file 1: Figure S1B and S2A, B). The tetra-linoleoyl $(m / z$ 1447.9) CL was oxidized in various species (i.e., $m / z$ 1463.9, 1479.9, 1495.9, and 1511.9 corresponding to the addition of 1 to 4 oxygens). In the heart sample of non-treated or doxorubicin-treated males, we could detect CLox; however, the level of these species were very low $(<0.5 \%$; Fig. $4 \mathrm{a}, \mathrm{b})$. Even if the percentage of $\mathrm{CL}+4[\mathrm{O}]$ and $\mathrm{CL}+$ $5[\mathrm{O}]$ appeared slightly more important in the DOXOtreated males than in the non-treated ones, the global level of oxidation seemed rather minimal. For this reason, we did not analyze the CLox in the female animals. Overall, these results suggest that oxidative damage to cardiac cardiolipin is not largely found after 7 weeks of doxorubicin treatment. These data are in accordance with our previous results showing that global protein carbonylation was comparable in the heart of males and females treated or not with doxorubicin [5].

\section{Cardiolipin biosynthesis is altered in males and females treated with doxorubicin}

CL biosynthesis is a multi-step process and the nascent CL from phosphatidyl glycerol (PG) is remodeled by different enzymes to form a "mature" CL (Additional file 1: Figure S3). Another important remodelling pathway includes the conversion of MLCL to CL. Moreover, a link has been made between the content of PL(18:2) and the poly-unsaturated FA metabolism [32]. Thus, we analyzed the PG and MLCL cardiac content. We identified only eight PG species (Additional file 1: Table S2 and Fig. 5a). In contrast to $\mathrm{PE}$ and $\mathrm{PC}$, we did not detect PG containing long acyl chains. However, similar to PE and PC, PG consisting of linoleoyl acyl chains (18:2) combined with saturated palmitic or stearic acyl chains did not present sex difference but were strongly reduced by doxorubicin treatment in both sexes (Fig. 5a). To note, PG (16:0-18:1) was specifically upregulated in doxorubicin-treated females. Concerning MLCL, we identified 26 different members (Additional file 1: Table S2) with the most abundant form:

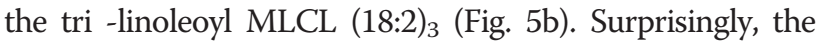
level of this MLCL did not vary between groups. However, similar to CL, doxorubicin induced a sex-specific remodelling of MLCL species with longer acyl chains (Fig. 5c).

Finally, the expression of key genes involved in phospholipid, FA metabolism, and CL remodelling was assessed (Fig. 6). No significant change was observed for the plasma membrane fatty acid transport protein Cd36, CDP-diacylglycerol synthase2 (Cds2), Acyl-CoA: lysocardiolipin acyltransferase-1 (Alcat1), and 45 -desaturase expression among the four groups. A lower expression of $C d s 1$ was observed in non-treated females compared to males. Doxorubicin induced a decrease in expression of acyl-CoA synthetase long-chain family member 1 Acsl1, Cds1, cardiolipin synthase1 (Crls1), Tafazzin, 46 -desaturase, and Elongase 5 for males. Regarding treated females, decreased expression was similar as males for $C d s 1$ and 


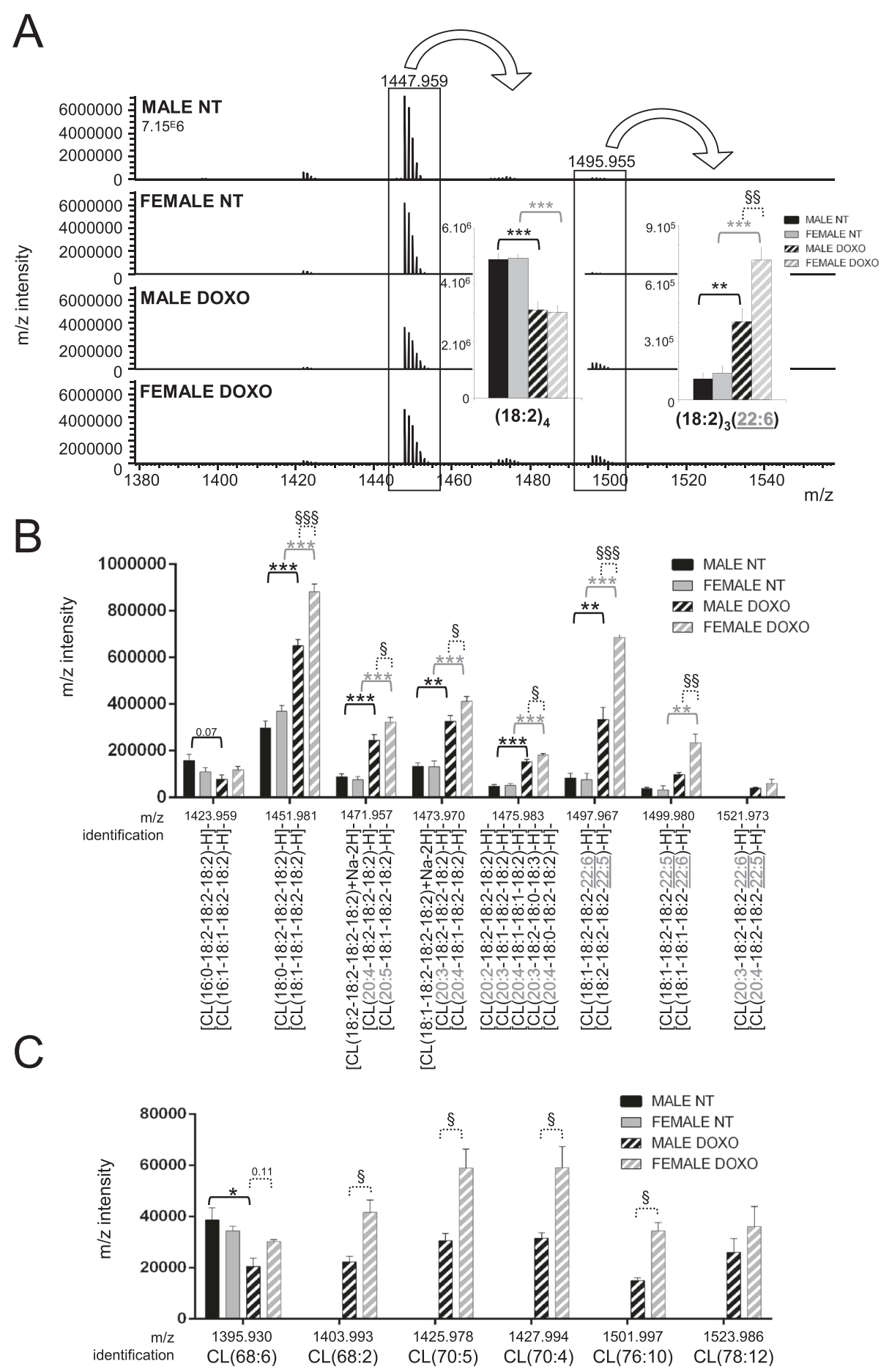

Fig. 3 Sex-specific cardiolipin remodelling after doxorubicin treatment. a Cardiolipin spectra obtained by LC-ESI/MS from male and female rats treated for 7 weeks with doxorubicin (DOXO) or saline (NT). Insert panels: mean intensity of $\mathrm{CL}(18: 2)_{4}$ and $\mathrm{CL}(18: 2)_{3}(22: 6)$. b Identified cardiolipin $\mathrm{m} / \mathrm{z}$ intensity (mean \pm SEM of four rats per group, duplicated samples). c Non-identified cardiolipin intensity mainly present in doxorubicin-treated rats 
Table 2 Statistical analysis of sex, doxorubicin treatment, and interaction between sex and treatment for the three main phospholipids

\begin{tabular}{|c|c|c|c|c|}
\hline $\mathrm{PL}-m / z$ & Identification & Sex effect & DOXO effect & $\begin{array}{c}\text { Sex and } \\
\text { DOXO } \\
\text { interaction }\end{array}$ \\
\hline CL 1497.967 & $(18: 1)(18: 2)_{2}(22: 6) ;(18: 2)_{3}(22: 5)$ & 0.005 & $<0.001$ & 0.004 \\
\hline CL 1499.980 & $(18: 1)(18: 2)_{2}(22: 5) ;(18: 1)_{2}(18: 2)(22: 6)$ & 0.046 & 0.002 & 0.035 \\
\hline CL 1495.956 & $(18: 2)_{3}(22: 6)$ & 0.008 & $<0.001$ & 0.022 \\
\hline CL 1451.981 & $(18: 0)(18: 2)_{3} ;(18: 1)_{2}(18: 2)_{2}$ & $<0.001$ & $<0.001$ & 0.017 \\
\hline PC 864.572 & $(16: 0-22: 6)$ & 0.002 & 0.018 & 0.76 \\
\hline PC 892.602 & $(18: 0-22: 6)$ & $<0.001$ & $<0.001$ & 0.95 \\
\hline PC 868.602 & $(18: 0-20: 4)$ & 0.033 & 0.06 & 0.70 \\
\hline PC 894.615 & $(18: 0-22: 5)$ & $<0.001$ & 0.30 & 0.46 \\
\hline PE 748.525 & (P-16:0-22:5); (P-18:1- 20:4) & $<0.001$ & 0.83 & 0.45 \\
\hline PE 762.504 & $(16: 0-22: 6)$ & $<0.001$ & 0.52 & 0.70 \\
\hline PE 792.546 & $(18: 0-22: 5)$ & $<0.001$ & 0.17 & 0.20 \\
\hline PE 790.534 & $(18: 0-22: 6)$ & $<0.001$ & 0.13 & 0.56 \\
\hline CL 1423.959 & $(16: 0)(18: 2)_{3} ;(16: 1)(18: 1)(18: 2)_{2}$ & 0.81 & 0.11 & 0.049 \\
\hline CL 1471.957 & $\begin{array}{l}(18: 2)_{4} \mathrm{Na} ;(18: 2)_{3}(20: 4) \\
(18: 2)_{1}(18: 2)_{2}(20: 5)\end{array}$ & 0.11 & $<0.001$ & 0.033 \\
\hline CL 1395.930 & $(68: 6)$ & 0.52 & 0.02 & 0.11 \\
\hline CL 1421.945 & $(18: 2)_{3}(16: 1)$ & 0.43 & $<0.001$ & 0.06 \\
\hline CL 1447.959 & $(18: 2)_{4}$ & 0.95 & $<0.001$ & 0.79 \\
\hline CL 1473.969 & $\begin{array}{l}(18: 1)(18: 2)_{3} \mathrm{Na} ;(18: 2)_{3}(20: 3) \\
(18: 2)_{2}(18: 1)(20: 4) \\
(18: 2)_{2}(20: 2) ;(18: 2){ }_{2}(18: 1)(20: 3)\end{array}$ & 0.08 & $<0.001$ & 0.07 \\
\hline CL 1475.983 & $\begin{array}{l}(18: 2)_{3}(20: 2) ;(18: 2)_{2}(18: 1)(20: 3) \\
(18: 2)(18: 1)_{2}(20: 4) ;(18: 2)_{2}(18: 0)(20: 4) \\
(18: 2)(18: 0)(18: 3)(20: 3)\end{array}$ & 0.08 & $<0.001$ & 0.14 \\
\hline PC 816.572 & $(16: 0-18: 2)$ & 0.22 & $<0.001$ & 0.96 \\
\hline PC 844.602 & $(18: 0-18: 2) ;(18: 1-18: 1)$ & 0.61 & $<0.001$ & 0.24 \\
\hline PE 714.505 & $(16: 0-18: 2)$ & 0.99 & $<0.001$ & 0.16 \\
\hline PE 742.536 & $(18: 0-18: 2) ;(18: 1-18: 1)$ & 0.48 & $<0.001$ & 0.67 \\
\hline
\end{tabular}

Crl1 and less important for the other genes, except for $\Delta 6$-desaturase and Elongase5 as no reduction was observed. Taken together, these results show a sex-specific remodelling of MLCL species with longer acyl chains in females and a more reduced expression of enzymes involved in fatty acid synthesis pathway in doxorubicintreated males than females (Additional file 1: Figure S3).

\section{Discussion}

In support of our previous work showing male-specific doxorubicin cardiotoxicity [5], this study describes for the first time the cardiac phospholipidome after doxorubicin treatment in both sexes. The role of phospholipids in the heart and the sex impact has been poorly explored, despite essential structure and signal transduction functions. After chronic doxorubicin treatment in male and female adult rats, we characterized doxorubicin and sex-specific phospholipid remodelling as best discriminating component by PCA.

From the 1980s, alterations of heart phospholipid content have been described in many cardiovascular diseases. Decrease in linoleic acid and increases in arachidonic and docosahexaenoic acid have been observed in myocardial phospholipids of rats and cats after TAC [33] and chronic coronary artery ligation [34]. A link has been made between alteration in PL and the severity of heart failure. PE, PC, PI, and PS, the main classes of PL, are present in different membranes of cardiomyocytes (sarcolemma, RS, mitochondria). These phospholipids are involved in various signal transduction pathways; they can be split by phospholipases into inositol triphosphate and diacylglycerol that function as second messengers. As we used heart tissue, we cannot discriminate the sarcolemma PL from the intracellular membrane organelles PL. Additional investigations are 


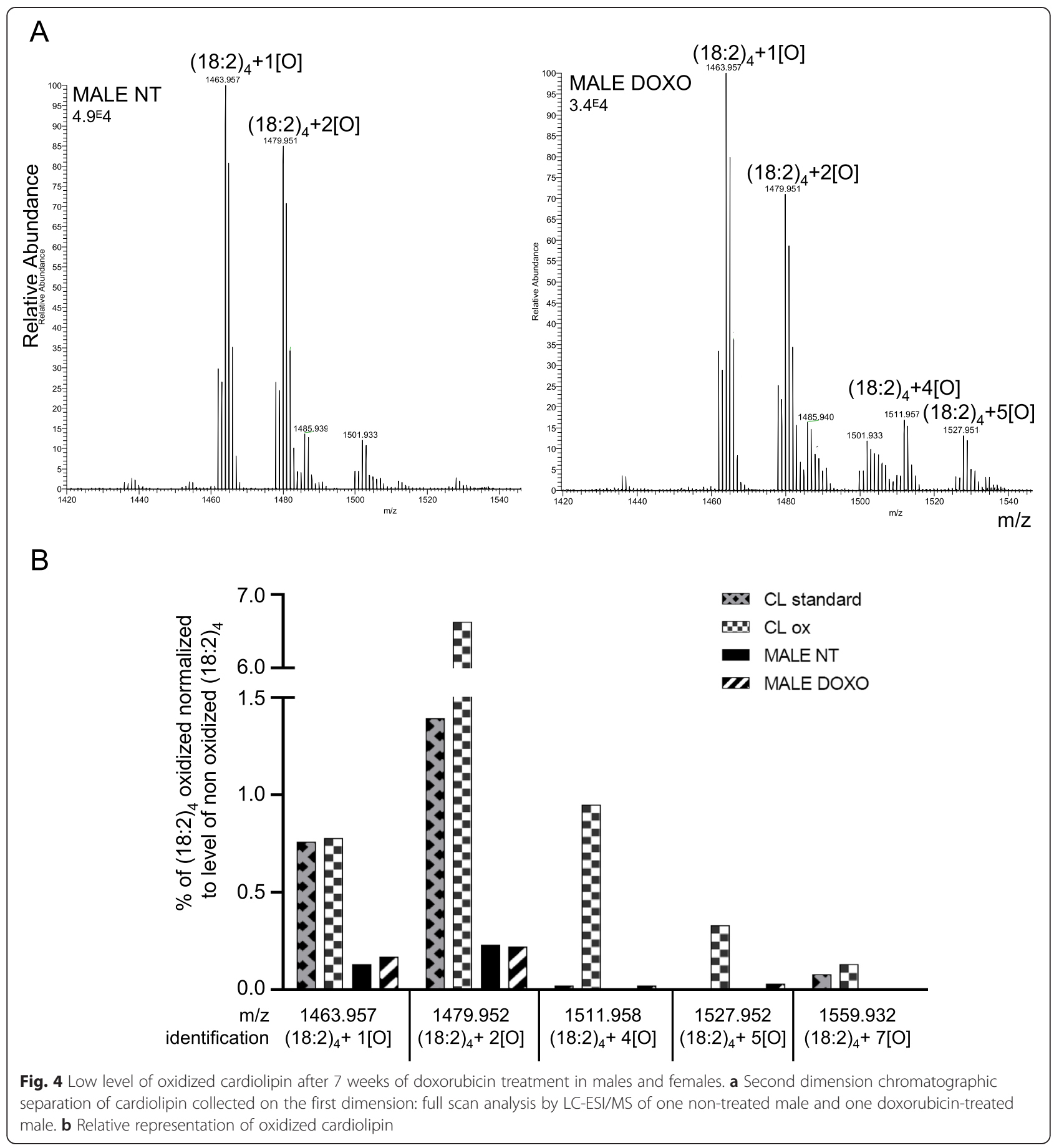

needed to determine the exact composition of the different membrane organelles. Thus, we will focus the discussion on CL because there are specific to the inner mitochondrial membrane and appears critical for mitochondrial function and cardiac pathology [9].

Several studies have highlighted the essential role of the mitochondria in heart failure and in anthracycline cardiotoxicity [5, 35-37]. The energetic depletion linked to mitochondrial defect is often related to a reduction in mitochondrial mass. However, when maximal oxygen consumption decreased and activities of key enzymes of energy metabolism (e.g., CS, CK, and COX) remained stable with no change in protein level of the peroxisome proliferator-activated receptor gamma co-activator 1 (PGC-1), the orchestrator for mitochondrial biogenesis, mitochondrial energetic deficiency has been suggested to be linked to a disruption of local environment [5, 38]. One key parameter for optimal and functional mitochondria is 


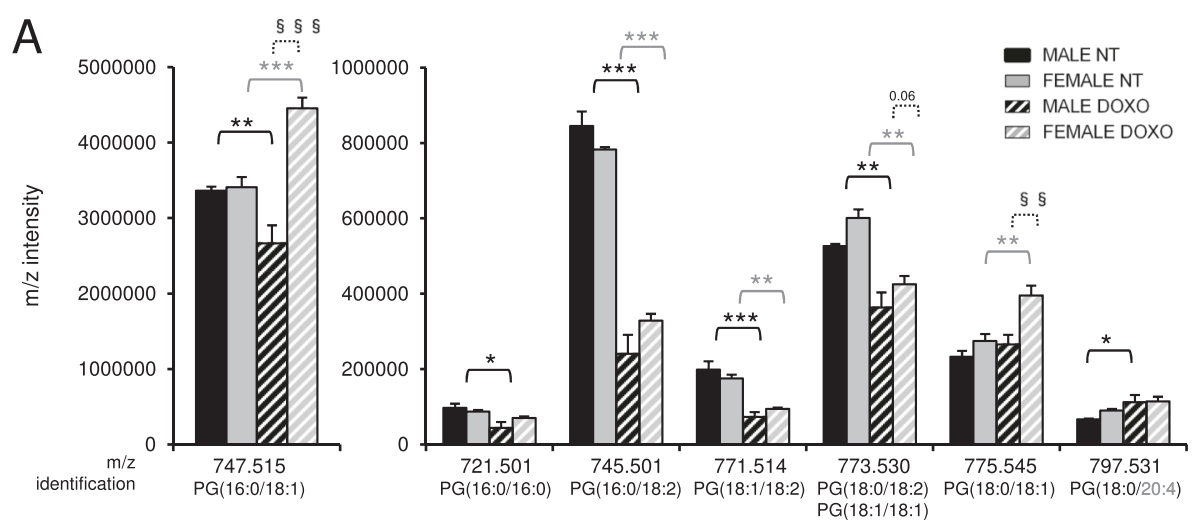

B

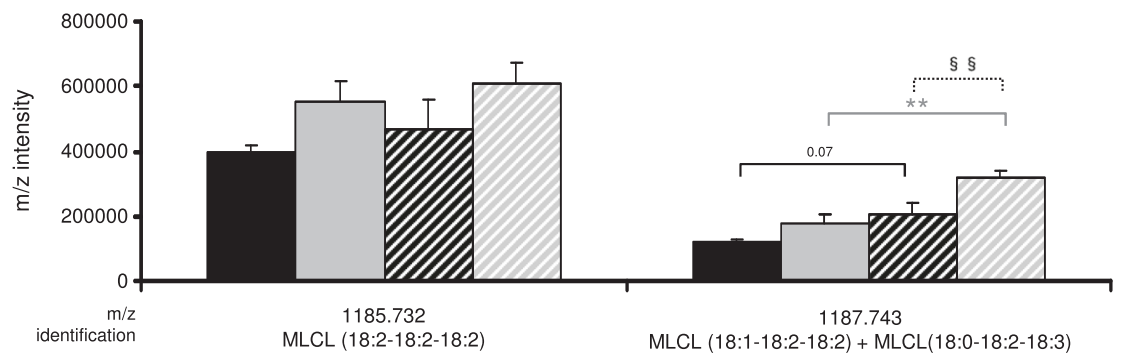

C

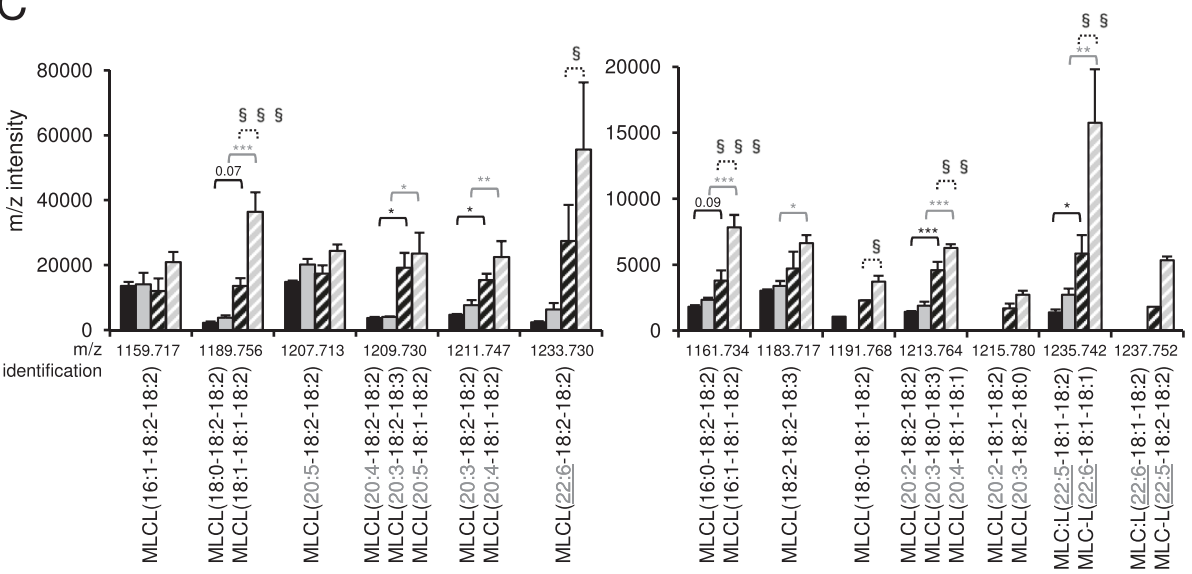

Fig. 5 Doxorubicin and sex effects on cardiac PG and MLCL profiles. a Analysis of $\mathrm{m} / \mathrm{z}$ intensity of PG species by LC-ESI/MS (mean \pm SEM of four rats per group, duplicated samples). b Analysis of $\mathrm{m} / \mathrm{z}$ intensity of MLCL species by LC-ESI/MS

cardiolipin. Not surprisingly, CL alterations such as content, distribution, oxidation, and acyl chain composition have been reported in many diseases including Barth syndrome, Parkinson disease, and heart failure [14]. As previously described [13], the main molecular species of cardiolipin is the $\mathrm{L}_{4} \mathrm{CL}(18: 2)_{4}$, representing around $55 \%$ of total CL in male and female rat hearts. This level was reduced to $35 \%$ in both sexes after doxorubicin treatment. Decrease of linoleic acid in cardiolipin has also been observed in ischemic, dilated, diabetic cardiomyopathies, and in senescent heart $[20,39,40]$. Moreover, most of the time, increase in 22:6 docosahexaenoic acid was noticed $[20,39,40]$. Hearts from doxorubicin-treated males presented an increase in CL containing 22:6 acyl chain, and this was highly exacerbated for the females. Moreover, some CL species appeared in DOXO-treated animals that were not present in controls also with a significantly higher content in females, indicating the occurrence of an alternate form of $\mathrm{CL}$ remodelling after DOXO treatment. Such a cardiac long acyl chain PL remodelling has been reported in heart failure and aging [32, 41]. One important point is that all these studies showing acyl chain CL remodelling were obtained in male animals/patients, and no comparison was made with females. This remodelling in males might be a compensatory mechanism but still insufficient to correct $\mathrm{CL}$ alterations. Because the total CL 


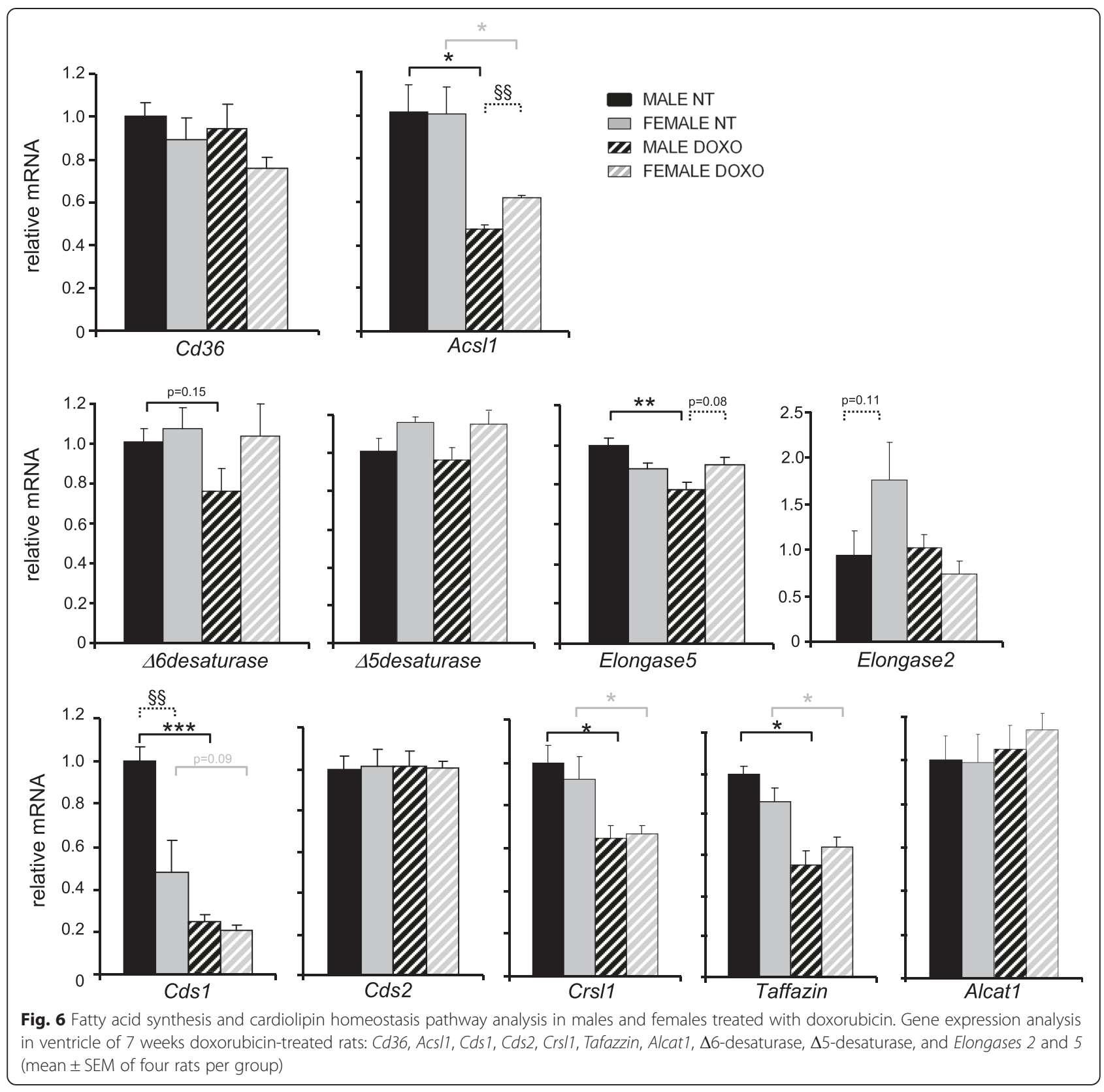

level was not reduced in DOXO-treated females and because mitochondrial function was not altered [5], it can be inferred that the significant increase in longer acyl chain (20:2, 20:3, 20:4, 22:5, and 22:6) could have compensated for the loss of $(18: 2)_{4}$ CL thus preserving the local environment for normal mitochondrial function. Indeed, calcium retention, cytochrome $\mathrm{c}$ sequestration, membrane fusion and fission, respiratory chain, and apoptosis are all dependent on CL content [9-12].

Because one of the controversial molecular mechanisms of anthracycline-induced cardiotoxicity is oxidative stress [42-44], we used a recently developed two-dimensional LC-MS/MS $[28,29]$ to analyze oxidized CL. Various conditions can cause cardiolipin oxidation, among them cytochrome $\mathrm{c} / \mathrm{H}_{2} \mathrm{O}_{2}$ and oxygenated $\mathrm{Fe}^{+}$buffer (hydroxyl radical generated by the Fenton and the Haber-Weiss) reactions [28, 31, 45]. As doxorubicin leads to accumulation of iron in the mitochondria [46], we used ferrous sulfate buffer for CLox-positive control. We did not observe a high level of CLox in doxorubicin-treated males. Indeed, around $0.05 \%$ of $(18: 2)_{4}$ containing 4 to 7 oxygens was detected. This result is in accordance with our previous data showing no obvious global protein oxidation [5]. Obviously, oxidative stress is species-, time-, and drug concentration-dependent, in other words model-dependent [42-44, 46-48]. It seems that while high level of CLox has 
been observed in acute stress, level of CLox could be moderate in chronic stress. After traumatic brain injury in postnatal day 17 rats, the content of oxidized CL species increased 20-fold at the expense of non-oxidized species [49]. Chronic western diet of LDLR-/- (low density lipoprotein receptor knockout) mice during 16 weeks induced a $0.1 \%$ significant increase of oxidized cardiolipin in the liver mitochondria [50]. Therefore, we can hypothesize that either chronic doxorubicin treatment with moderate doses induces only low levels of CLox or that CLox may be produced early after injection but rapidly discarded to avoid mitochondrial dysfunction.

In DOXO-treated males but not females, we observed an early decrease in PGC- $1 \beta$ [5]. Recently, PGC- $1 \alpha / \beta$, key transcriptional regulators of mitochondrial metabolism, has been implicated in the regulation of cardiac phospholipid biosynthesis [51]. Despite grossly normal cardiac function of PGC- $1 \alpha / \beta$ heart-/-, mitochondrial ultrastructure abnormalities were observed [51]. PGC$1 \alpha / \beta$ hearts $-/$ - were characterized by normal level of MLCL but reduced levels of most PE, PC, and CL and a decreased level of CDP-diacylglycerol synthase Cds-1 showing that $C d s-1$ was a direct target of PGC-1. We observed that Cds-1 was highly downregulated after doxorubicin in both sexes and even in basal level for females. In PGC- $1 \alpha / \beta$ heart $-/-$, a reduction in PLcontaining 22:5 and 22:6 acyl chains was observed [51] whereas in our model an increase was noticed. Thus, PL remodelling in DOXO cardiotoxicity can hardly be explained by PGC-1 downregulation.

It has been proposed that remodelling of $\mathrm{CL}$ can be linked to poly-unsaturated fatty acid metabolism [32]. An interesting feature of female cardiac PLs is the presence of species with long acyl chains not only in CL but in other PL classes. Elevated long-chain omega 3 fatty acids in females under the same diet than males have been reported in numerous studies [52]. This sex difference has been linked in part to estrogens, FA remodelling enzymes, and peroxisome proliferator-activated receptor alpha (PPAR $\alpha)$ activity. In the liver, but not the heart, gene expression of $46-$ and 45 -desaturases and Elongases, enzymes involved in the production of long-chain polyunsaturated FAs from 18:2, is greater in females [53]. In our study, supply of essential fatty acid ( $\alpha$-linolenic acid 18:3 and linoleic acid 18:2) from diet was the same for males and females treated or not with doxorubicin. However, in males, the specific decrease of Elongase5 and the tendency to decrease for $\Delta 6$-desaturase were more suggestive of impaired rather than improved production of long-chain fatty acids from $\alpha$-linolenic acid 18:3 and linoleic acid 18:2. Expression of these enzymes has been shown to be under the control of an estrogen-mediated PPAR a activation. Interestingly, we have previously observed that PPAR $\alpha$ was less downregulated in doxorubicin-treated females [5]. Regarding FA pathway, the gene expression of $C d 36$, a regulator of the first step, i.e., uptake of FA across the plasma membrane, remained stable after DOXO in both sexes. However, Acsl1, the major acyl-CoA synthethase isoform in the heart that converts long-chain fatty acid into acyl-CoA thioesters plus AMP was also less downregulated in females. Interestingly, ACSL1 in the liver was described to play an important role in directing FA into pathways of phospholipid synthesis and away from cholesterolesterification and $\beta$ oxidation [54]. After doxorubicin treatment, we have shown that multiple steps of $\beta$-oxidation (MCAD and CPT1) were downregulated similarly in both sexes [5]. It appeared that FA pathway of PL synthesis rather than $\beta$ oxidation seems more preserved in females that have a better cardiac function (Additional file 1: Figure S3). In line with all these results, we have previously observed that females treated with doxorubicin have intensified liver hepatomegaly and elevated circulating level of triglycerides compared to males [5]. Similar to our results, Julicher et al. also observed a more important hepatomegaly after doxorubicin treatment in females [55]. We can hypothesize that a protective mechanism linked to lipid metabolism is activated only in females.

Finally, sex difference in cardiotoxicity of doxorubicin has not been extensively studied. However, some clinical and experimental studies have observed that females develop less unwanted cardiac side effects after doxorubicin treatment than males $[5,55-59]$. The mechanism of female cardioprotection is far from being completely understood. Among the proposed pathways involved were higher oxidative defenses capacity in females [57], increased level of cardiac mast cells activity in males [59], and better mitochondrial metabolism and bioenergetic protection in females [5]. Interestingly, interactions between doxorubicin and negatively charged phospholipid and more specifically cardiolipin have been demonstrated by several groups [2, 4]. Cardiolipins are essential for respiratory chain functioning, ATP generation, and energy transfer. The heart is a highly energy-consuming organ, and cardiac contraction is linearly related to mitochondrial respiration thus anthracycline-mediated cardiolipin alterations would be a determinant factor in mitochondrial alterations and cardiotoxicity [5, 37, 44, 60]. We have now shown that cardiolipin remodelling is probably important in the sexual dimorphism of doxorubicininduced cardiotoxicity. Additional studies are necessary to better understand the roles of lipids in the anthracycline cardiotoxicity and sex differences.

\section{Conclusions}

In this study, we showed that (1) a decrease of global phospholipids was only observed in doxorubicin-treated males, (2) the level of PE and PC species containing long 
acyl chains was more important in females than in males with or without doxorubicin, (3) doxorubicin mediated a decline of PE and PC containing one linoleoyl acyl chain in both sexes, (4) significant loss of $\mathrm{L}_{4} \mathrm{CL}$ was comparable in treated males and females while (18:2) 3 MLCL remained stable, (5) the level of most long-chain CL and MLCL species was more important specifically for doxorubicin-treated females, (6) CL oxidation did not appear to be a key parameter in this chronic treatment, and (7) sex differences were found in gene expression of FA biosynthesis after doxorubicin treatment. Altogether, the cardiac phospholipidomic analysis has highlighted an interesting sex-specific PL molecular species remodelling (Table 2).

This study has unraveled a significant sexual dimorphism in the cardiac phospholipid content after a chronic cardiotoxic stress. A cause-and-effect relationship between altered phospholipid content and sex differences in doxorubicin-induced cardiotoxicity remains to be established. Further studies are needed to decode the male-specific cardiotoxicity to be able to develop new therapeutic strategies.

\section{Additional file}

Additional file 1: Expanded method section. Figure S1: Detection of oxidized cardiolipin. Figure S2: LC-MS/MS analysis of tetralinoleoyl cardiolipin with 1 to 4 oxygens. Figure S3: Speculative model of sex-specific cardiac cardiolipin remodelling after doxorubicin treatment. (PDF $171 \mathrm{~kb}$ )

\section{Abbreviations}

CLs: cardiolipins; HF: heart failure; $\mathrm{L}_{4} \mathrm{CL}$ : tetra-linoleoyl cardiolipin (18:2) ${ }_{4}$; LC: liquid chromatography; MLCL: monolysocardiolipin; MS: mass spectrometry; PC: phosphatidylcholine; PE: phosphatidylethanolamine; PG: phosphatidylglycerol; PI: phosphatidylinositol; PL: phospholipid; PS: phosphatidylserine; ROS: reactive oxygen species.

\section{Competing interests}

The authors declare that they have no competing interests.

\section{Authors' contributions}

MM performed most of the experimental work on rats. AS performed lipidomics analysis. W and AG provided critical comments. MM, RVC, and PC designed, directed the experiments, and wrote the manuscript. All authors read and approved the final manuscript.

\section{Acknowledgements}

We thank Valérie Domergue and Pauline Robert (Animal facility of UMS IPSIT) for their help with the animals and Claudine Deloménie (Transcriptomic platform of UMS IPSIT) for her expertise in molecular biology. We thank Philippe Mateo and Jean-Louis Paul for helpful discussion, Frédéric Joubert for critical reading, and Rodolphe Fischmeister for continuous support.

\section{Funding sources}

Our laboratory is a member of the Laboratory of Excellence LERMIT. This work is supported by a grant from the "Fondation pour la Recherche Médicale" (DPM2012-125,554), Université Paris-Sud (ERM), and CORDDIM and MALINF-DIM from Région Ile de France. MM is recipient of a fellowship from the "Fondation ARC." RVC is an emeritus scientist at CNRS.

\section{Author details}

'UMR-S 1180, Inserm, Univ Paris-Sud, Université Paris-Saclay,

Châtenay-Malabry, France. ${ }^{2}$ SFR IPSIT (Institut Paris-Saclay d'Innovation Thérapeutique), Univ Paris-Sud, Université Paris-Saclay, Châtenay-Malabry, France. ${ }^{3}$ UMS IPSIT Service d'Analyse des Médicaments et Métabolites, Châtenay-Malabry, France. ${ }^{4}$ Lip(Sys)2 ex EA4041, Univ Paris-Sud, Université Paris-Saclay, Châtenay-Malabry, France. ${ }^{5}$ Current address: Université Paris Diderot, Unité de Biologie Fonctionnelle et Adaptative, CNRS UMR 8251, Paris, France.

Received: 14 July 2015 Accepted: 6 October 2015

Published online: 15 October 2015

\section{References}

1. Mordente A, Meucci E, Silvestrini A, Martorana GE, Giardina B. Anthracyclines and mitochondria. Adv Exp Med Biol. 2012;942:385-419 doi:10.1007/978-94-007-2869-1_18.

2. Parker MA, King V, Howard KP. Nuclear magnetic resonance study of doxorubicin binding to cardiolipin containing magnetically oriented phospholipid bilayers. Biochim Biophys Acta. 2001;1514(2):206-16.

3. Tokarska-Schlattner M, Dolder M, Gerber I, Speer O, Wallimann T, Schlattner U. Reduced creatine-stimulated respiration in doxorubicin challenged mitochondria: particular sensitivity of the heart. Biochim Biophys Acta. 2007;1767(11):1276-84.

4. Goormaghtigh E, Huart P, Praet M, Brasseur R, Ruysschaert JM. Structure of the adriamycin-cardiolipin complex. Role in mitochondrial toxicity. Biophys Chem. 1990;35(2-3):247-57.

5. Moulin M, Piquereau J, Mateo P, Fortin D, Rucker-Martin C, Gressette M, et al. Sexual dimorphism of doxorubicin-mediated cardiotoxicity: potential role of energy metabolism remodeling. Circulation. 2015;8:98-108. doi:10.1161/CIRCHEARTFAILURE.114.001180.

6. Dessi M, Noce A, Bertucci P, Manca di Villahermosa S, Zenobi R, Castagnola V, et al. Atherosclerosis, dyslipidemia, and inflammation: the significant role of polyunsaturated fatty acids. ISRN inflammation. 2013;2013:191823. doi:10.1155/ 2013/191823.

7. Kullenberg D, Taylor LA, Schneider M, Massing U. Health effects of dietary phospholipids. Lipids Health Dis. 2012;11:3. doi:10.1186/1476-511X-11-3.

8. Bogdanov M, Mileykovskaya $E$, Dowhan W. Lipids in the assembly of membrane proteins and organization of protein supercomplexes: implications for lipid-linked disorders. Subcell Biochem. 2008;49:197-239. doi:10.1007/978-1-4020-8831-5_8.

9. Houtkooper $\mathrm{RH}$, Vaz FM. Cardiolipin, the heart of mitochondrial metabolism. Cell Mol Life Sci. 2008;65(16):2493-506. doi:10.1007/s00018-008-8030-5.

10. Mejia EM, Cole LK, Hatch GM. Cardiolipin metabolism and the role it plays in heart failure and mitochondrial supercomplex formation. Cardiovasc Hematol Disord Drug Targets. 2014;14(2):98-106.

11. Paradies G, Paradies V, De Benedictis V, Ruggiero FM, Petrosillo G. Functional role of cardiolipin in mitochondrial bioenergetics. Biochim Biophys Acta. 2014;1837(4):408-17. doi:10.1016/j.bbabio.2013.10.006.

12. Ye C, Shen Z, Greenberg ML. Cardiolipin remodeling: a regulatory hub for modulating cardiolipin metabolism and function. Journal of bioenergetics and biomembranes. 2014. doi: 10.1007/s10863-014-9591-7.

13. Schlame M, Brody S, Hostetler KY. Mitochondrial cardiolipin in diverse eukaryotes. Comparison of biosynthetic reactions and molecular acyl species. Eur J Biochem/FEBS. 1993;212(3):727-35.

14. Chicco AJ, Sparagna GC. Role of cardiolipin alterations in mitochondrial dysfunction and disease. Am J Physiol. 2007;292(1):C33-44. doi:10.1152/ ajpcell.00243.2006

15. Ingwall JS. Energy metabolism in heart failure and remodelling. Cardiovasc Res. 2009;81(3):412-9.

16. Neubauer $\mathrm{S}$. The failing heart-an engine out of fuel. N Engl J Med. 2007;356(11):1140-51.

17. Ventura-Clapier R, Garnier A, Veksler V. Transcriptional control of mitochondrial biogenesis: the central role of PGC-1alpha. Cardiovasc Res. 2008;79(2):208-17.

18. Saini-Chohan HK, Holmes MG, Chicco AJ, Taylor WA, Moore RL, McCune SA, et al. Cardiolipin biosynthesis and remodeling enzymes are altered during development of heart failure. J Lipid Res. 2009;50(8):1600-8. doi:10.1194/ jIr.M800561-JLR200

19. Sparagna GC, Chicco AJ, Murphy RC, Bristow MR, Johnson CA, Rees ML, et al. Loss of cardiac tetralinoleoyl cardiolipin in human and experimental heart failure. J Lipid Res. 2007;48(7):1559-70. doi:10.1194/jlr.M600551-JLR200. 
20. Han X, Yang J, Cheng H, Yang K, Abendschein DR, Gross RW. Shotgun lipidomics identifies cardiolipin depletion in diabetic myocardium linking altered substrate utilization with mitochondrial dysfunction. Biochemistry. 2005:44(50):16684-94. doi:10.1021/bi051908a.

21. Schlame M, Towbin JA, Heerdt PM, Jehle R, DiMauro S, Blanck TJ. Deficiency of tetralinoleoyl-cardiolipin in Barth syndrome. Ann Neurol. 2002;51(5):634-7. doi:10.1002/ana.10176.

22. Lightfoot JC, D'Agostino Jr RB, Hamilton CA, Jordan J, Torti FM, Kock ND, et al. Novel approach to early detection of doxorubicin cardiotoxicity by gadolinium-enhanced cardiovascular magnetic resonance imaging in an experimental model. Circ Cardiovasc Imaging. 2010;3(5):550-8.

23. Dworatzek E, Mahmoodzadeh S, Schubert C, Westphal C, Leber J, Kusch A, et al. Sex differences in exercise-induced physiological myocardial hypertrophy are modulated by oestrogen receptor beta. Cardiovasc Res. 2014;102(3):418-28. doi:10.1093/cvr/cvu065.

24. Folch J, Lees M, Sloane Stanley GH. A simple method for the isolation and purification of total lipides from animal tissues. J Biol Chem 1957;226(1):497-509.

25. Paradies G, Ruggiero FM, Petrosillo G, Quagliariello E. Age-dependent decline in the cytochrome c oxidase activity in rat heart mitochondria: role of cardiolipin. FEBS Lett. 1997:406(1-2):136-8.

26. Imbert L, Ramos RG, Libong D, Abreu S, Loiseau PM, Chaminade P. Identification of phospholipid species affected by miltefosine action in Leishmania donovani cultures using LC-ELSD, LC-ESI/MS, and multivariate data analysis. Anal Bioanal Chem. 2012;402(3):1169-82. doi:10.1007/s00216-011-5520-3.

27. Pulfer M, Murphy RC. Electrospray mass spectrometry of phospholipids. Mass Spectrom Rev. 2003;22(5):332-64. doi:10.1002/mas.10061.

28. Kim J, Minkler PE, Salomon RG, Anderson VE, Hoppel CL. Cardiolipin: characterization of distinct oxidized molecular species. J Lipid Res. 2011;52(1):125-35. doi:10.1194/jlr.M010520.

29. Tyurin VA, Tyurina YY, Jung MY, Tungekar MA, Wasserloos KJ, Bayir $H_{\text {, }}$ et al. Mass-spectrometric analysis of hydroperoxy- and hydroxyderivatives of cardiolipin and phosphatidylserine in cells and tissues induced by pro-apoptotic and pro-inflammatory stimuli. J Chromatogr B Analyt Technol Biomed Life Sci. 2009;877(26):2863-72. doi:10.1016/ j.jchromb.2009.03.007

30. Pluskal T, Castillo S, Villar-Briones A, Oresic M. MZmine 2: modular framework for processing, visualizing, and analyzing mass spectrometry-based molecular profile data. BMC bioinformatics. 2010;11:395. doi:10.1186/1471-2105-11-395.

31. Samhan-Arias AK, Ji J, Demidova OM, Sparvero LJ, Feng W, Tyurin V, et al. Oxidized phospholipids as biomarkers of tissue and cell damage with a focus on cardiolipin. Biochim Biophys Acta. 2012;1818(10):2413-23. doi:10.1016/j.bbamem.2012.03.014.

32. Le CH, Mulligan CM, Routh MA, Bouma GJ, Frye MA, Jeckel KM, et al. Delta6-desaturase links polyunsaturated fatty acid metabolism with phospholipid remodeling and disease progression in heart failure. Circulation. 2014;7(1):172-83. doi:10.1161/CIRCHEARTFAILURE.113.000744.

33. Reibel DK, O'Rourke B, Foster KA, Hutchinson H, Uboh CE, Kent RL. Altered phospholipid metabolism in pressure-overload hypertrophied hearts. Am J Physiol. 1986;250(1 Pt 2):H1-6.

34. Nasa Y, Sakamoto $Y$, Sanbe A, Sasaki H, Yamaguchi F, Takeo S. Changes in fatty acid compositions of myocardial lipids in rats with heart failure following myocardial infarction. Mol Cell Biochem. 1997;176(1-2):179-89.

35. Garnier A, Fortin D, Delomenie C, Momken I, Veksler V, Ventura-Clapier R. Depressed mitochondrial transcription factors and oxidative capacity in rat failing cardiac and skeletal muscles. J Physiol. 2003;551(Pt 2):491-501.

36. Garnier A, Fortin D, Zoll J, N'Guessan B, Mettauer B, Lampert E, et al. Coordinated changes in mitochondrial function and biogenesis in healthy and diseased human skeletal muscle. Faseb J. 2005;19(1):43-52.

37. Tokarska-Schlattner M, Wallimann T, Schlattner U. Alterations in myocardial energy metabolism induced by the anti-cancer drug doxorubicin. C R Biol. 2006;329(9):657-68.

38. Athea Y, Viollet B, Mateo P, Rousseau D, Novotova M, Garnier A, et al. AMPactivated protein kinase alpha2 deficiency affects cardiac cardiolipin homeostasis and mitochondrial function. Diabetes. 2007;56(3):786-94.

39. Heerdt PM, Schlame M, Jehle R, Barbone A, Burkhoff D, Blanck TJ. Diseasespecific remodeling of cardiac mitochondria after a left ventricular assist device. Ann Thorac Surg. 2002;73(4):1216-21.

40. Lee HJ, Mayette J, Rapoport SI, Bazinet RP. Selective remodeling of cardiolipin fatty acids in the aged rat heart. Lipids Health Dis. 2006;5:2. doi:10.1186/1476-511X-5-2.
41. Mulligan $\mathrm{CM}$, Le $\mathrm{CH}$, deMooy AB, Nelson CB, Chicco AJ. Inhibition of delta- 6 desaturase reverses cardiolipin remodeling and prevents contractile dysfunction in the aged mouse heart without altering mitochondrial respiratory function. J Gerontol A Biol Sci Med Sci. 2014;69(7):799-809. doi:10.1093/gerona/glt209.

42. Menna P, Paz OG, Chello M, Covino E, Salvatorelli E, Minotti G. Anthracycline cardiotoxicity. Expert Opin Drug Saf. 2012;11 Suppl 1:S21-36.

43. Octavia Y, Tocchetti CG, Gabrielson KL, Janssens S, Crijns HJ, Moens AL Doxorubicin-induced cardiomyopathy: from molecular mechanisms to therapeutic strategies. J Mol Cell Cardiol. 2012;52(6):1213-25.

44. Sterba M, Popelova O, Vavrova A, Jirkovsky E, Kovarikova P, Gersl V, et al Oxidative stress, redox signaling and metal chelation in anthracycline cardiotoxicity and pharmacological cardioprotection. Antioxid Redox Signal. 2013;18:899-929.

45. Paradies G, Ruggiero FM. Age-related changes in the activity of the pyruvate carrier and in the lipid composition in rat-heart mitochondria. Biochim Biophys Acta. 1990;1016(2):207-12.

46. Ichikawa Y, Ghanefar M, Bayeva M, Wu R, Khechaduri A, Naga Prasad SV, et al. Cardiotoxicity of doxorubicin is mediated through mitochondrial iron accumulation. J Clin Invest. 2014;124(2):617-30. doi:10.1172/JCI72931.

47. Jirkovsky E, Popelova O, Krivakova-Stankova P, Vavrova A, Hroch M, Haskova $P$, et al. Chronic anthracycline cardiotoxicity: molecular and functional analysis with focus on Nrf2 and mitochondrial biogenesis pathways. J Pharmacol Exp Ther. 2012;343:468-78.

48. Aryal B, Jeong J, Rao VA. Doxorubicin-induced carbonylation and degradation of cardiac myosin binding protein C promote cardiotoxicity. Proc Natl Acad Sci U S A. 2014;111(5):2011-6. doi:10.1073/pnas.1321783111.

49. Ji J, Kline AE, Amoscato A, Samhan-Arias AK, Sparvero LJ, Tyurin VA, et al. Lipidomics identifies cardiolipin oxidation as a mitochondrial target for redox therapy of brain injury. Nat Neurosci. 2012;15(10):1407-13. doi:10.1038/nn.3195.

50. Zhong H, Lu J, Xia L, Zhu M, Yin H. Formation of electrophilic oxidation products from mitochondrial cardiolipin in vitro and in vivo in the context of apoptosis and atherosclerosis. Redox biology. 2014;2:878-83. doi:10.1016/ j.redox.2014.04.003.

51. Lai L, Wang M, Martin OJ, Leone TC, Vega RB, Han X, et al. A role for peroxisome proliferator-activated receptor gamma coactivator 1 (PGC-1) in the regulation of cardiac mitochondrial phospholipid biosynthesis. J Biol Chem. 2014;289(4):2250-9. doi:10.1074/jbc.M113.523654.

52. Kitson AP, Stroud CK, Stark KD. Elevated production of docosahexaenoic acid in females: potential molecular mechanisms. Lipids. 2010;45(3):209-24. doi:10.1007/s11745-010-3391-6.

53. Kitson AP, Smith TL, Marks KA, Stark KD. Tissue-specific sex differences in docosahexaenoic acid and Delta-6 desaturase in rats fed a standard chow diet. Appl Physiol Nutr Metab = Physiologie appliquee, nutrition et metabolisme. 2012;37(6):1200-11. doi:10.1139/h2012-103.

54. Li LO, Mashek DG, An J, Doughman SD, Newgard CB, Coleman RA. Overexpression of rat long chain acyl-CoA synthetase 1 alters fatty acid metabolism in rat primary hepatocytes. J Biol Chem. 2006;281(48):37246-55. doi:10.1074/jbc.M604427200.

55. Julicher RH, Sterrenberg L, Haenen GR, Bast A, Noordhoek J. The effect of chronic adriamycin treatment on heart kidney and liver tissue of male and female rat. Arch Toxicol. 1988;61(4):275-81.

56. Hequet $\mathrm{O}$, Le QH, Moullet I, Pauli E, Salles G, Espinouse D, et al. Subclinical late cardiomyopathy after doxorubicin therapy for lymphoma in adults. J Clin Oncol. 2004;22(10):1864-71.

57. Julicher RH, Sterrenberg L, Haenen GR, Bast A, Noordhoek J. Sex differences in the cellular defence system against free radicals from oxygen or drug metabolites in rat. Arch Toxicol. 1984;56(2):83-6.

58. van Hoesel QG, Steerenberg PA, Dormans JA, de Jong WH, de Wildt DJ, Vos JG. Time-course study on doxorubicin-induced nephropathy and cardiomyopathy in male and female LOU/M/Wsl rats: lack of evidence for a causal relationship. J Natl Cancer Inst. 1986;76(2):299-307.

59. Zhang J, Knapton A, Lipshultz SE, Cochran TR, Hiraragi H, Herman EH. Sexrelated differences in mast cell activity and doxorubicin toxicity: a study in spontaneously hypertensive rats. Toxicol Pathol. 2014;42(2):361-75. doi:10.1177/0192623313482778. Epub 2013 Mar 26.

60. Ventura-Clapier R, Garnier A, Veksler V, Joubert F. Bioenergetics of the failing heart. Biochim Biophys Acta. 2011;1813(7):1360-72. 